\title{
Stabilization of the Peregrine soliton and Kuznetsov-Ma breathers by means of nonlinearity and dispersion management
}

\author{
J. Cuevas-Maraver \\ Grupo de Física No Lineal, Departamento de Física Aplicada I, Universidad de Sevilla. Escuela Politécnica \\ Superior, C/ Virgen de África, 7, 41011-Sevilla, Spain \\ Instituto de Matemáticas de la Universidad de Sevilla (IMUS). Edificio Celestino Mutis. Avda. Reina Mercedes s/n, \\ 41012-Sevilla, Spain \\ Boris A. Malomed \\ Department of Physical Electronics, School of Electrical Engineering, Faculty of Engineering, \\ Tel Aviv University, Tel Aviv 69978, Israel \\ P. G. Kevrekidis \\ Department of of Mathematics and Statistics, University of Massachusetts, \\ Amherst, MA 01003-9305, USA \\ D. J. Frantzeskakis \\ Department of Physics, National and Kapodistrian University of Athens, Panepistimiopolis, Zografos, Athens 15784, \\ Greece
}

\begin{abstract}
We demonstrate a possibility to make rogue waves (RWs) in the form of the Peregrine soliton (PS) and Kuznetsov-Ma breathers (KMBs) effectively stable objects, with the help of properly defined dispersion or nonlinearity management applied to the continuous-wave $(\mathrm{CW})$ background supporting the RWs. In particular, it is found that either management scheme, if applied along the longitudinal coordinate, making the underlying nonlinear Schrödinger equation (NLSE) selfdefocusing in the course of disappearance of the PS, indeed stabilizes the global solution with respect to the modulational instability of the background. In the process, additional excitations are generated, namely, dispersive shock waves and, in some cases, also a pair of slowly separating dark solitons. Further, the nonlinearity-management format, which makes the NLSE defocusing outside of a finite domain in the transverse direction, enables the stabilization of the KMBs, in the form of confined oscillating states. On the other hand, a nonlinearity-management format applied periodically along the propagation direction, creates expanding patterns featuring multiplication of KMBs through their cascading fission.
\end{abstract}

Keywords:

Nonlinear Schrödinger equation; rogue waves; modulational instability; dispersive shock waves; dark solitons 


\section{Introduction}

The nonlinear Schrödinger equation (NLSE) and its variants are well known as universal models for nonlinear waves and solitons, as well as relevant phenomenology, in many areas of physics including water waves, plasmas, nonlinear optics, Bose-Einstein condensates, and so on. [1, 2, 3, 4, 5, 6, 7, 8, 9]. Among various solutions of these equations, a class of unstable but physically meaningful ones represent rogue waves (RWs), which can spontaneously emerge on top of continuous-wave (CW) modulationally (alias Benjamin-Feir [10, 11]) unstable states, and then disappear. RWs were originally identified in terms of water waves in the ocean [12]. Later, this concept was extended to nonlinear fiber optics [13, 14, 15, 16, 17] and other areas (see, e.g., Refs. [18, 19, 20, 21]). Recently, the pioneering work of [22] argued that the so-called Peregrine solitons (PSs) are a generic byproduct of a phenomenon called gradient catastrophe arising at the level of the semi-classical form of the NLSE. Moreover such solutions also emerged in the context of interactions of dispersive shock waves [23]. An overview of the current state of the studies of RWs can be found in Ref. [24, 25].

The classical integrable NLSE with the cubic self-focusing nonlinearity, in terms of the spatialdomain propagation (or with the anomalous group-velocity dispersion (GVD), in terms of fiber optics [2]) gives rise both to the CW states subject to the modulational instability, and to exact RW solutions, the most fundamental ones being the Peregrine soliton (PS) [26], the KuznetsovMa breather (KMB) [27, 28], and the Akhmediev breather [29]. The PS is a state of an instanton type built on top of the CW background, i.e., it is localized both in the longitudinal and transverse coordinates (if the NLSE is considered as a model of a planar waveguide in the spatial domain). The KMB, on the other hand, is localized in the transverse direction, and periodically oscillate in the longitudinal one, while the Akhmediev breather [29], is periodic in the transverse direction and self-localized along the propagation distance. Due to the fact that all these states are supported by the modulationally unstable background, they are unstable too, which poses a limitation to their physical realizations; even when they are carefully realized experimentally [17], the modulational instability of the background cannot be avoided. On the other hand, the concept of the dispersion and nonlinearity management [30,6] suggests a possibility to stabilize RWs by making the GVD and/or local nonlinearity coefficients functions of the propagation distance or transverse coordinate. This way, these solitons and breathers would have enough room to emerge in areas where the NLSE is self-focusing, and, on the other hand, the background may be globally stabilized by making the NLSE self-defocusing outside of the area reserved for the formation of the RWs. The objective of the present work is to demonstrate the "proof of principle" as regards these possibilities for the effective stabilization of the PS and KMBs, applying the schemes of both the dispersion and nonlinearity management. While our focus here is on numerical experiments, the existence [30] and earlier experimental implementation [30, 31] of related schemes suggests their potential consideration in (near-)future optical and related physical systems.

The paper is organized as follows. The model and numerical methods used for its analysis are presented in Section II. The results obtained for the stabilization of the PS and KMBs, under the action of the management, are reported, respectively, in Sections III and IV (while both the dispersion and nonlinearity management are applied to the PS, only the latter scheme is considered for the KMBs). Finally, the paper is concluded by Section V. 


\section{The model and numerical scheme}

The NLSE which we use for the stabilization of the PSs and KMBs is taken as

$$
i u_{z}+\frac{1}{2} D(z) u_{x x}+\gamma(x, z)|u|^{2} u=0 .
$$

In the spatial domain, which corresponds to the light propagation in a planar waveguide, the diffraction coefficient is constant, $D(z) \equiv 1$, while the local nonlinearity coefficient may be modulated as a function of the propagation and transverse coordinates, $z$ and $x$ [6]. In the temporal domain, corresponding to the light propagation in an optical fiber, $x$ is actually the reduced time, $\tau \equiv t-z / V_{\mathrm{gr}}$ ( $t$ is time proper, and $V_{\mathrm{gr}}$ is the group velocity of the carrier wave), the relevant fiber's model has $\gamma(x, z) \equiv 1$, while the GVD coefficient, $D(z)$ may be made a function of the propagation length, using known techniques of the GVD management [30, 15].

The integrable version of the NLSE, i.e., Eq. (1) with $D(z) \equiv 1$ and $\gamma(x, z) \equiv 1$, gives rise to the exact PS [26] and KMB [27, 28] solutions:

$$
\begin{gathered}
u_{\mathrm{PS}}(x, z)=\left[1-\frac{4(1+2 \mathrm{i} z)}{1+4 x^{2}+4 z^{2}}\right] \mathrm{e}^{\mathrm{i} z} . \\
u_{\mathrm{KMB}}(x, z)=\left[1+\frac{2(1-2 a) \cos (\omega z)-\mathrm{i} \omega \sin (\omega z)}{\sqrt{2 a} \cosh (b x)-\cos (\omega z)}\right] \mathrm{e}^{\mathrm{i} z},
\end{gathered}
$$

where $a \equiv\left(1+\sqrt{\omega^{2}+1}\right) / 4$ and $b \equiv 2 \sqrt{2 a-1}$, while $\omega$ is an arbitrary frequency of the KMB oscillations. As explained in the Introduction, both solutions are supported by the CW background, $\exp (i z)$, which is prone to the modulational instability.

To demonstrate effects of management, we present here results of numerical simulations of Eq. (1) with initial condition:

$$
u(x, 0)=u_{\mathrm{PS}}\left(x, z_{0}\right), z_{0}=-5,
$$

when dealing with PS (the choice of $z_{0}=-5$ is appropriate for demonstrating both the growth and the decay phase of the wave structure). In the case of KMBs, the input is taken as:

$$
u(x, 0)=u_{\mathrm{KMB}}(x, 0) .
$$

In the latter case, we set $\omega=1.5$ here, as this value was found to be appropriate for representing the generic situation. Note that, as RW solutions possess relatively steep peaks, the present version of the NLSE is a mildly stiff equation for simulations, in these cases. To handle it, we have used the exponential time differencing fourth-order Runge-Kutta numerical algorithm [33]. The discretization of the second derivative was performed by dint of the Fourier spectral collocation, implying periodic boundary conditions imposed on the integration domain, $-L<x<+L$. Here we report results produced for $L=200$, and a discretization spacing $\Delta x=25 / 256 \approx 0.10$, as well as a time step $\Delta t=(\Delta x)^{2} / 4$. These parameters ensure the stability of the numerical integration.

Figure 1 shows the outcome of the simulations performed for the NLSE (1) in the absence of management, $D=\gamma \equiv 1$, using the above-mentioned PS and KMB wave forms as initial 

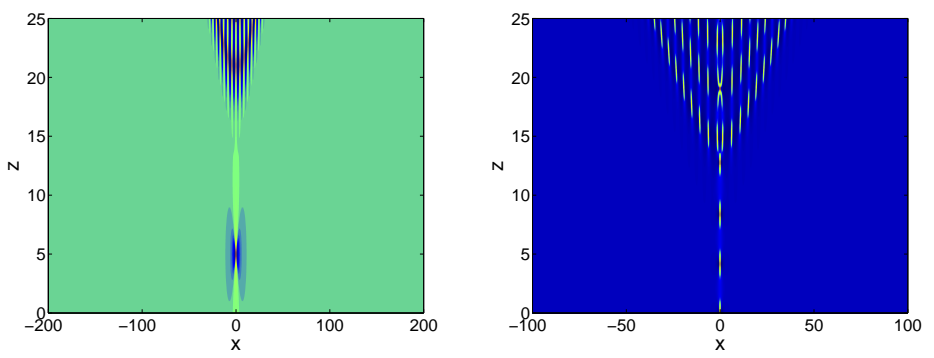

Figure 1: Density plots illustrating the evolution of a Peregrine soliton (left) and a Kuznetsov-Ma breather with $\omega=1.5$ (right) in the framework of the constant-coefficient NLSE (1), which does not include any management.

conditions. The onset of the modulational instability, seeded by truncation errors of the numerical algorithm, is clearly observed at the center of the domain. It is natural that this occurs there, as the presence of the PS amplifies growing perturbations on top of the unstable background. Notice that, recently, the instability of the KMB -and by extension of the PS in the limit of vanishing frequency- was analyzed via Floquet theory in Ref. [34].

\section{The management of Peregrine solitons}

First, we test the effects of the management applied to the PS. For this purpose, we have performed simulations of Eq. (1) with either $D \equiv 1$ and $z$-dependent nonlinearity $\gamma(z)$, or vice versa. As we show below, in both cases outcomes are quite similar. The nonlinearity management is implemented as:

$$
\begin{aligned}
\gamma(x, z) & =\left\{\begin{array}{ll}
1 & \text { at } z<z_{1} \\
-1 & \text { at } z \geq z_{1}
\end{array},\right. \\
D & \equiv 1,
\end{aligned}
$$

i.e., the originally focusing nonlinearity switches to defocusing at $z=z_{1}$, while the dispersion management can be introduced as

$$
\begin{aligned}
D(z) & =\left\{\begin{array}{ll}
1 & \text { for } z<z_{1} \\
-1 & \text { for } z \geq z_{1}
\end{array},\right. \\
\gamma(x, z) & \equiv 1 .
\end{aligned}
$$

In the latter case, the nonlinearity keeps the focusing sign, while the GVD changes from anomalous to normal at $z=z_{1}$. As said above, the results shown here correspond to the PS launched by means of input (4).

Typical examples demonstrating the application of the nonlinearity and dispersion management to the PS are displayed in Fig. 2, It is observed that, as expected, the modulational instability is suppressed, and the main additional excitations arising past the disappearance of the PS are the dispersive shock waves (cf. Refs. [35, 36, 37, 38, 39, 40] and for a recent review Ref. [41]) propagating on top of the uniform background (the modulationally stable one, due to the adopted management format). Remarkably, at $z_{1}=-z_{0}=5$, a pair of dark solitons is formed too. 

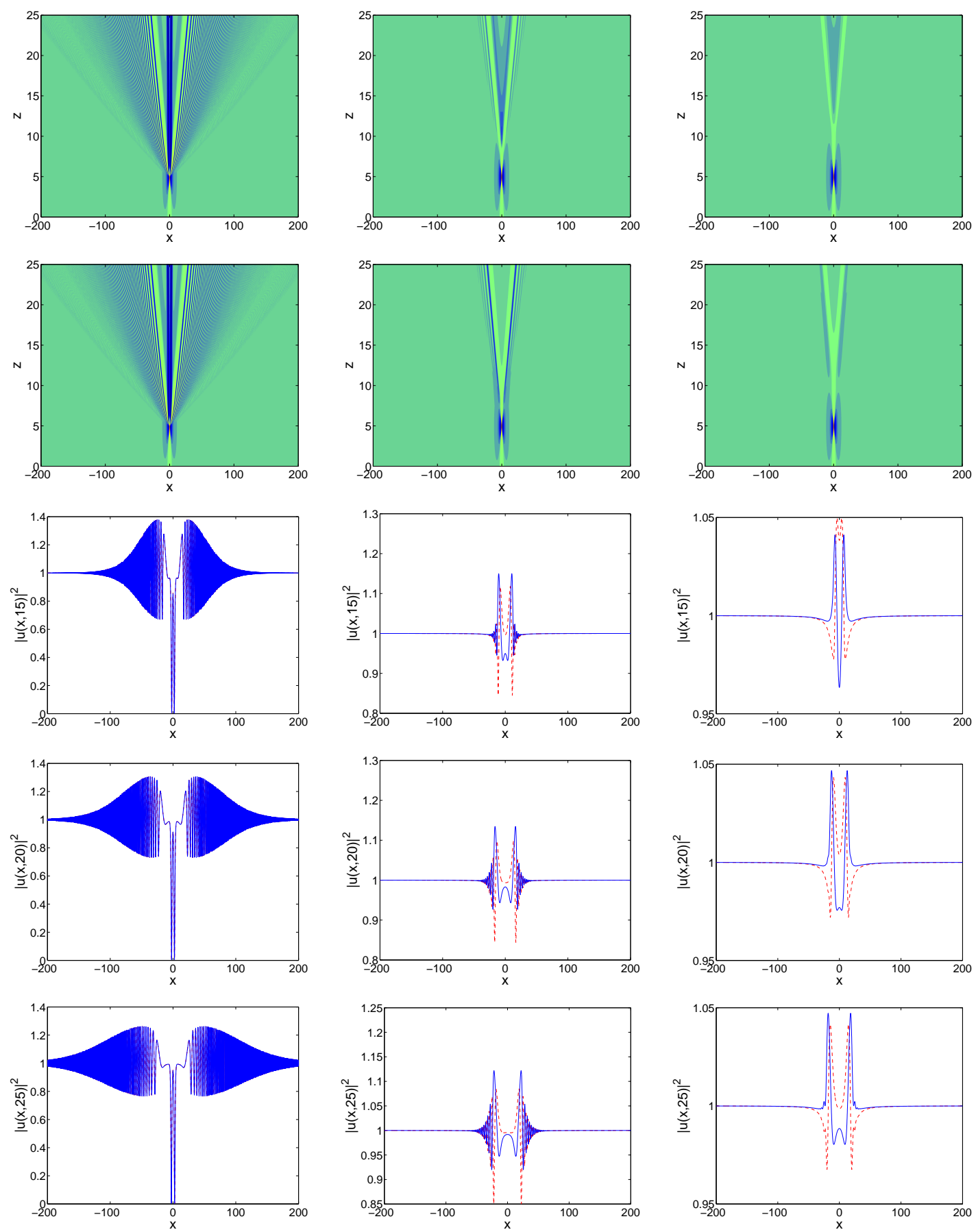

Figure 2: Evolution of Peregrine solitons under the action of the management, with $z_{1}=-z_{0}=5$ (left panels), $z_{1}=7.5$ (central panels) and $z_{1}=10$ (right panels). Panels in the first and second row display density plots under the action of the nonlinearity and dispersion management, see Eqs. (6) and (7), respectively. Other panels show snapshots of the managed Peregrine soliton at different values of $z$, with continuous blue and dashed red lines corresponding, respectively, to the nonlinearity and dispersion management. 
These dark solitons separate slowly because the repulsive interaction between them is weak, being screened by the shock-wave pattern. For larger values of $z_{1}$, dark solitons do not emerge; instead, there appear a pair of central dips, whose depth quickly decreases with $z_{1}$. The depth of the dip is related to that of the exact PS (2) at $z=z_{1}$.

Finally, we note that similar dynamical scenarios are observed under the action of the nonlinearity and dispersion managements. Differences between these two management schemes, which increase with $z_{1}$, amount to quantitative (yet no major qualitative) details.

\section{The management of Kuznetsov-Ma breathers}

Given the similarity of dispersion and nonlinearity management for the PSs, in the case of KMBs, we have systematically studied only the nonlinearity management, fixing $D(z) \equiv 1$ in NLSE (1). We have considered two different management formats. One of them acts only along the transverse coordinate, $x$, without dependence on the propagation coordinate $(z)$ :

$$
\gamma(x, z)= \begin{cases}1 & \text { for }|x|<x_{1} \\ -1 & \text { for }|x| \geq x_{1}\end{cases}
$$

that is, the nonlinearity is focusing at $|x|<x_{1}$ and defocusing at $|x| \geq x_{1}$. The other format acts periodically along $z$ (in accordance with the fact that the exact KMB solution (3) is a periodic function if $z$ ), being independent of $x$ :

$$
\gamma(x, z)=\cos (\omega z) .
$$

Generic examples of the numerical results produced by formats (8) and (9) are displayed in Fig. 3. In the former case, with $x_{1}=2$, we observe the establishment of a robust confined pattern with a regular breathing shape and a gradually growing amplitude (although its spatial extent appears to be slightly decreasing). For the same management format (8), but with $x_{1}=15$, the simulations produce a persistent confined pattern (within the region of action of the management) with a more complex structure. Its distinct feature is the presence of individual large amplitude events (within the domain of focusing nonlinearity) emerging and disappearing in quick succession in a way reminiscent of PSs.

The $z$-periodic management format (9) produces a completely different picture, as seen in the right column of Fig. 3, the seed breather undergoes initial splitting, which is followed by a cascade of splittings, and systematic expansion of the area occupied by the multi-breather pattern. Here, the large amplitude events are less "ordered" in their emergence (and are less transparently persistent at $x=0$ ), yet they still appear to be present in the short-intermediate scale dynamics monitored herein.

\section{Conclusion}

In conclusion, we demonstrated the possibility to make rogue waves (RWs) stable objects in NLSE models, avoiding the modulational instability of the backgrounds on top of which they arise. This was achieved by applying the appropriately designed schemes of the dispersion and 

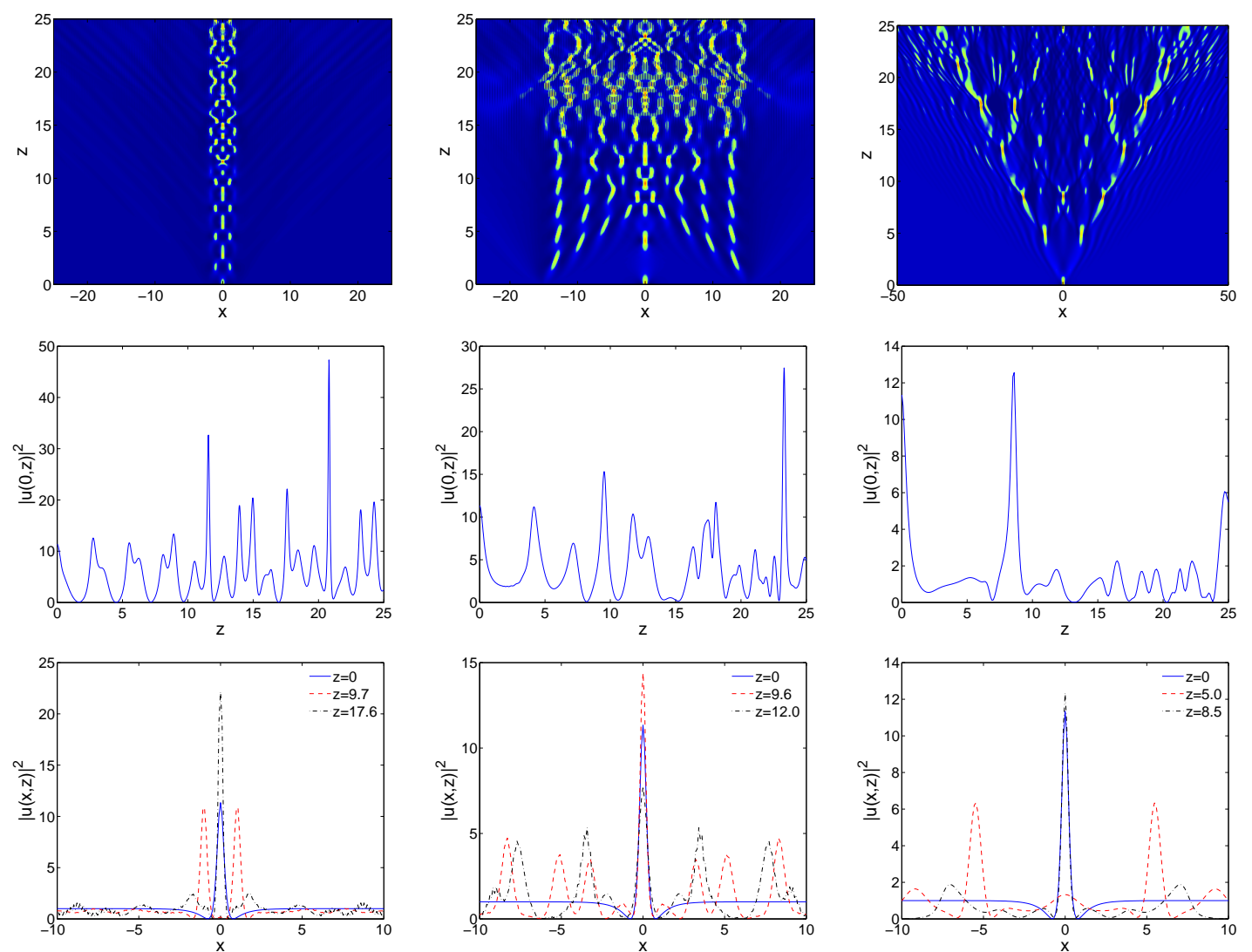

Figure 3: Evolution of Kuznetsov-Ma breathers under the action of the nonlinearity management in the format (8), for $x_{1}=2$ and $x_{1}=15$ (left and central columns, respectively), and in the format (9) (right column). The top panels display density plots of the breathers. The middle panels represent the evolution of the density at $x=0$. The bottom panels show profiles of the breathers at different values of $z$. 
nonlinearity management to the CW background supporting the RWs in the form of the Peregrine soliton (PS) and Kuznetsov-Ma breathers (KMBs). In particular, it was found that both types of management, applied along the propagation distance, indeed stabilize the PS, generating, after its disappearance, additional dynamically persistent features, in the form of dispersive shock waves and, sometimes, an additional pair of slowly separating dark solitons. On the other hand, the nonlinearity management, which makes the NLSE defocusing outside of a finite domain in the transverse direction, stabilizes the KMBs in the form of robustly propagating confined breatherlike states, while the nonlinearity management applied periodically along the propagation direction gives rise to expanding patterns driven by cascading fissions of the breathers.

As further development of the analysis, it may be interesting to consider interactions of two or several PSs in the framework of the present models, based on the dispersion and nonlinearity management. Other extensions of this work include the investigation of interactions of PSs with defects or their consideration in higher dimensions (under stabilized backgrounds). These directions are presently under consideration and will be reported accordingly in future studies.

\section{Acknowledgements.}

J. C.-M. thanks financial support from MAT2016-79866-R project (AEI/FEDER,UE). P.G.K. gratefully acknowledges the support of NSF-PHY-1602994, the Alexander von Humboldt Foundation, the Stavros Niarchos Foundation via the Greek Diaspora Fellowship Program, and the ERC under FP7, Marie Curie Actions, People, International Research Staff Exchange Scheme (IRSES605096). The work of B.A.M. is supported, in part, by the joint program in physics between NSF and Binational (US-Israel) Science Foundation through project No. 2015616, and by the Israel Science Foundation through Grant No. 1286/17. This author also appreciates a grant provided by the European Erasmus Plus program for visiting the National and Kapodistrian University of Athens (Greece). P.G.K and D.J.F. acknowledge that this work made possible by NPRP grant \#[8-764-160] from Qatar National Research Fund (a member of Qatar Foundation). The findings achieved herein are solely the responsibility of the authors.

\section{References}

[1] J. Ablowitz and P. A. Clarkson, Solitons, Nonlinear Evolution Equations and Inverse Scattering (Cambridge University Press, Cambridge, 1990).

[2] G. P. Agrawal, Nonlinear Fiber Optics (Academic Press: San Diego, 1995).

[3] L. Pitaevskii and S. Stringari, Bose-Einstein Condensation (Oxford University Press, Oxford, 2003).

[4] B. A. Malomed, D. Mihalache, F. Wise, and L. Torner, J. Opt. B 7, R53-R72 (2005); J. Phys. B: At. Mol. Opt. Phys. 49, 170502 (2016).

[5] R. Carretero-González, D. J. Frantzeskakis, and P. G. Kevrekidis, Nonlinearity 21, R139 (2008).

[6] Y. V. Kartashov, B. A. Malomed, and L. Torner, Solitons in nonlinear lattices, Rev. Mod. Phys. 83, 247-306 (2011).

[7] V. S. Bagnato, D. J. Frantzeskakis, P. G. Kevrekidis, B. A. Malomed, and D. Mihalache, Rom. Rep. Phys. 67, 5 (2015).

[8] B. A. Malomed, Eur. Phys. J. Spec. Topics 225, 2507 (2016).

[9] D. Mihalache, Rom. Rep. Phys. 69, 403 (2017).

[10] T. B. Benjamin and J. E. Feir, J. Fluid Mech. 27, 417 (1967).

[11] K. Dysthe, H. E. Krogstad, and P. Müller, Ann. Rev. Fluid Mech. 40, 287 (2008). 
[12] C. Kharif, E. Pelinovsky, and A. Slunyaev, Rogue Waves in the Ocean (Springer, Heidelberg, 2009).

[13] D. R. Solli, C. Ropers, P. Koonath, and B. Jalali, Nature 450, 1054 (2007).

[14] D. R. Solli, G. Herink, B. Jalali, and C. Ropers, Nature Photon. 6, 463 (2012).

[15] N. Akhmediev, J. M. Dudley, D. R. Solli, and S. K. Turitsyn, J. Opt. 15, 060201 (2013).

[16] J. M. Dudley, F. Dias, M. Erkintalo, and G. Genty, Nature Phys. 8, 755 (2014).

[17] A. Tikan, C. Billet, G. El, A. Tovbis, M. Bertola, T. Sylvestre, F. Gustave, S. Randoux, G. Genty, P. Suret, and J.M. Dudley Phys. Rev. Lett. 119, 033901 (2017).

[18] Yu. V. Bludov, V. V. Konotop, and N. Akhmediev, Phys. Rev. A 80, 033610 (2009).

[19] N. Akhmediev, A. Ankiewicz, and M. Taki, Phys. Lett. A 373, 675 (2009).

[20] N. Akhmediev and E. Pelinovsky, Eur. Phys. J. Special Topics 185, 1 (2010).

[21] Z. Y. Yan, Phys. Lett. A 374, 672 (2010); ibid. 375, 4274 (2011).

[22] M. Bertola and A. Tovbis, Comm. Pure Appl. Math. 66, 678 (2013).

[23] G. El, E.G. Khamis, A. Tovbis, Nonlinearity 29, 2798 (2016).

[24] N. Akhmediev, B. Kibler, F. Baronio, M. Belić, W.-P. Zhong, Y. Zhang, W. Chang, J. M. Soto-Crespo, P. Vouzas, P. Grelu, C. Lecaplain, K. Hammani, S. Rica, A. Picozzi, M. Tlidi, K. Panajotov, A. Mussot, A. Bendahmane, P. Szriftgiser, G. Genty, J. Dudley, A. Kudlinski, A. Demircan, U. Morgner, S. Amiramashvili, C. Bree, G. Steinmeyer, C. Masoller, N. G. R. Broderick, A. F. J. Runge, M. Erkintalo, S. Residori, U. Bortolozzo, F. T. Arecchi, S. Wabnitz, C. G. Tiofack, S. Coulibaly, and M. Taki, J. Opt. 18, 063001 (2016).

[25] S. Chen, F. Baronio, J. M. Soto-Crespo, P. Grelu, and D. Mihalache, J. Phys. A: Math. Theor. 50, 463001 (2017).

[26] D. H. Peregrine, J. Austral. Math. Soc. Ser. B (Appl. Math.) 25, 16 (1983).

[27] E. A. Kuznetsov, Sov. Phys.-Dokl. 22, 507 (1977).

[28] Y. C. Ma, Stud. Appl. Math. 60, 43 (1979).

[29] N. Akhmediev and V. I. Korneev, Theor. Math. Phys. 69, 1089 (1986).

[30] B. A. Malomed, Soliton Management in Periodic Systems (Springer: New York, 2006).

[31] M. Centurion, M.A. Porter, P.G. Kevrekidis, D. Psaltis, Phys. Rev. Lett. 97, 033903 (2006); M. Centurion, M.A. Porter, Y. Pu, P.G. Kevrekidis, D.J. Frantzeskakis, D. Psaltis, Phys. Rev. Lett. 97, 234101 (2006).

[32] S. K. Turitsyn, B. G. Bale, and M. P. Fedoruk, Dispersion-managed solitons in fibre systems and lasers, Phys. Rep. 521, 135-203 (2012).

[33] A.-K. Kassam and L. N. Trefethen, SIAM J. Sci. Comput. 26, 1214 (2005).

[34] J. Cuevas-Maraver, P. G. Kevrekidis, D. J. Frantzeskakis, N. I. Karachalios, M. Haragus, and G. James Phys. Rev. E 96, 012202 (2017)

[35] T. Y. Hou and P. D. Lax, Dispersive approximation in fluid dynamics, Commun. Pure Appl. Math. 44, 1-40 (1991).

[36] G. P. Agrawal and C. Headley, Kink solitons and optical shocks in dispersive nonlinear media, Phys. Rev. A 46, 1573-1577 (1992).

[37] M. A. Hoefer, M. J. Ablowitz, I. Coddington, E. A. Cornell, P. Engels, and V. Schweikhard, Dispersive and classical shock waves in Bose-Einstein condensates and gas dynamics, Phys. Rev. A 74, 023623 (2006).

[38] W. Wan, S. Shu, and J. W. Fleischer, Dispersive superfluid-like shock waves in nonlinear optics, Nature Phys. 3, 46-51 (2007).

[39] G. A. El, A. Gammal, E. G. Khamis, R. A. Kraenkel, and A. M. Kamchatnov, Theory of optical dispersive shock waves in photorefractive media, Phys. Rev. A 76, 053813 (2007).

[40] C. Conti, A. Fratalocchi, M. Peccianti, G. Ruocco, and S. Trillo, Observation of a gradient catastrophe generating solitons, Phys. Rev. Lett. 102, 083902 (2009).

[41] G.A. El, M.A. Hoefer, Physica D 333, 11 (2016). 